\title{
The Moscow Long-Term Program of Cepheid Radial Velocities
}

\author{
Nikolai Samus \\ Institute of Astronomy, Russian Acad. Sci., 48, Pyatnitskaya Str., \\ Moscow 109017, Russia and Sternberg Astronomical Institute \\ Natalia Gorynya \\ Institute of Astronomy, Russian Acad. Sci., 48, Pyatnitskaya Str., \\ Moscow 109017, Russia
}

\begin{abstract}
A correlation spectrometer has been used for 15 years by Moscow observers for different programs at several small telescopes and has proven itself to be very effective. Here we present some of the results in the field of Cepheid studies acquired with the spectrometer.
\end{abstract}

\section{Introduction}

We present some results of our program based upon observations of Galactic Cepheids with a CORAVEL-type correlation spectrometer. This program seems a good example of using the principal advantage of small-telescope programs, namely the possibility to have plenty of observing time over the course of many years, combined with special advantages of this particular instrument, namely its outstanding effectiveness combined with quite modest requirements in terms of size which allows it to be carried from one observing site to another, according to availability of telescopes and to weather conditions.

The spectrometer used, ILS, was designed and built by Tokovinin (1987) in 1986. It used the ideas first suggested by Felgett (1953) and by Griffin (1967) which were then implemented for echelle spectrometers in the CORAVEL machine (Baranne et al. 1979). In a CORAVEL-type spectrometer, an echelle spectrograph forms an image of a star's spectrum. A physical mask is placed in the focal plane. It is actually an image of the spectrum of a "standard" star, in our case, of Arcturus; the positions of the mask that correspond to spectral lines are transparent, while those between them are not. A special plane mirror added into the optical scheme oscillates at a frequency of about $10 \mathrm{~Hz}$ so that the observed spectrum moves back and forth along the mask. The flux passing through the mask is minimal when the lines of the observed spectrum coincide with the "lines" of the mask. The light is then collected and measured with a photomultiplier. A special controller (in the earlier configuration of the instrument) or a computer (currently) serves to collect the measurements of light separately for 50 time intervals of each oscillation cycle of the plane mirror. Thus, we obtain a "generalized spectral line", actually corresponding, in our case, to $\sim 1500$ lines in the spectrum of the program star or of Arcturus. The registered "line" covers a range of approximately $\pm 25 \mathrm{~km} / \mathrm{s}$, but we can search for the radial velocity in a much wider interval, about \pm 300 to $500 \mathrm{~km} / \mathrm{s}$, using calibrated rotation of 
the diffraction grating of the spectrometer. The generalized line is then fitted to a relevant profile (usually Gaussian), and the position of its minimum determines the radial velocity. Preliminary reductions are performed already during observations, but more sophisticated software can be used off-line to improve the accuracy. The reductions take into account all the necessary corrections for the motion of the observer; the zero point correction, usual for such instruments, is determined using observations of radial velocity standards. The profile shape gives additional information about abundances or rotation.

The main technical characteristics of the ILS are the following: It can measure radial velocities of main-sequence stars with "normal" chemical abundances in the spectral type range approximately from F5 to M5; somewhat earlier giants can also be measured. The characteristic accuracy for sufficiently bright stars in the middle of the spectral type range is 0.3 to $0.5 \mathrm{~km} / \mathrm{s}$. The limiting magnitude for telescopes of the $1-\mathrm{m}$ class is about $12^{m} .5$; in record cases, stars as faint as $14^{m}$ could be measured. The typical exposure time for brighter stars is about $5 \mathrm{~min}$; for faint stars, it seldom exceeds $30 \mathrm{~min}$.

Several instruments of CORAVEL-type design were built in the 1980s or early 1990s in different countries. Now, there are only few survivors. Surely there are advantages in registering the complete spectrum with a CCD and then determining the radial velocity (and numerous other parameters!) by means of purely digital reductions. However, instruments like the ILS have proved to be excellent machines for "the poor", the reductions are very simple, almost no additional technical support is needed, and you can bring your instrument to an isolated observatory and start observing the next night. We are going to continue the use of the ILS for several more years.

\section{Observations and Some Results}

During 15 years of active exploitation of the ILS, it has been used by several groups for many scientific programs, among them: orbits of binary and multiple stars; kinematics of the Galaxy; kinematics of stars in open and globular clusters; pulsations of stars (Cepheids and some others). Here we discuss only the last program.

In 1987-2000, we acquired more than 7000 observations of 144 Cepheids using 11 telescopes in 6 countries (Russia, Ukraine, Georgia, Azerbaijan, Uzbekistan, Bulgaria), having $60 \mathrm{~cm}$ to $2 \mathrm{~m}$ apertures. Table 1 shows some additional information. Primarily, telescopes of the $1-m$ class were used. Our main instrument was the $1 \mathrm{~m}$ telescope of the Simeiz Observatory (Crimea, Ukraine). The observatory is now a department of the Crimean Astrophysical Observatory, but, despite the disintegration of the Soviet Union, the telescope still belongs to the Institute of Astronomy, Moscow. Note that we use, rather effectively, the $70 \mathrm{~cm}$ telescope of the Sternberg Astronomical Institute, installed in Moscow, located less than $10 \mathrm{~km}$ from the Kremlin!

The specific feature of our program is that we try to obtain a good coverage of the pulsation velocity curve for each Cepheid during each year season. Our database of original accurate radial velocity measurements for Cepheids currently appears to be the world's richest. 
Table 1. Telescopes and Observations of Cepheids

\begin{tabular}{rlcr}
\hline No & Telescope & Years & No. of observ. \\
\hline 1 & $70 \mathrm{~cm}$, Moscow, Russia & $1987-2000$ & 867 \\
2 & $60 \mathrm{~cm}$, Nauchny, Crimea, Ukraine & $1987-1990$ & 47 \\
3 & $200 \mathrm{~cm}$, Shemakha, Azerbaijan & 1988 & 2 \\
4 & $122 \mathrm{~cm}$, Abastumani, Georgia & 1988 & 8 \\
5 & $125 \mathrm{~cm}$, Nauchny, Crimea, Ukraine & $1989-1990$ & 64 \\
6 & $100 \mathrm{~cm}$, Mt. Maidanak, Uzbekistan & $1989-1993$ & 283 \\
7 & $100 \mathrm{~cm}$, Simeiz, Crimea, Ukraine & $1990-2000$ & 3792 \\
8 & $60 \mathrm{~cm}$, Simeiz, Crimea, Ukraine & $1990-1998$ & 1902 \\
9 & $200 \mathrm{~cm}$, Mt. Rozhen, Bulgaria & 1990 & 16 \\
10 & $60 \mathrm{~cm}$, Mt. Maidanak, Uzbekistan & 1991 & 212 \\
11 & $60 \mathrm{~cm}$, Zvenigorod, Russia & 1997 & 18 \\
\hline \hline
\end{tabular}

Grand total: 14 years, 1341 nights, 7211 observations of 144 Cepheids

Figure 1 shows the characteristic radial velocity curves for Cepheids, folded with their pulsation periods. Note that the scatter of data points for X Cyg is very low, and that the velocity curve looks not worse than good photoelectric light curves of Cepheids. Thus, if the Baade-Wesselink technique is applied to determine Cepheid radii, the factor that crucially limits the accuracy is no longer the uncertainty in radial velocities.

The other two examples do not look so nice. If the period of the Cepheid is correct and does not vary strongly, there can be two reasons for the increased scatter. The first of them are double mode pulsations. We have observed several double-mode Cepheids and were able to separate the two pulsation modes in their radial velocities. EW Sct is a well-known double-mode Cepheid, we have more than 100 data points for it. Another interesting example of a double mode Cepheid in our program is V458 Sct, recently discovered by Antipin (1997). For this star, the first overtone amplitude is larger than that of the fundamental tone (Antipin et al. 1999); the same effect is revealed by the star's light curve, but the interpretation of the radial velocity curve in terms of energy is more straightforward, and the coexistence of two oscillations, with the first-overtone having a higher energy, poses a problem to the theory of stellar pulsations.

The second reason for the increased scatter is binarity. TX Del is an extreme case (it is not clear if the star is a classical Cepheid or a Population II Cepheid). We independently discovered the binarity of this star, first noted by Harris and Welch (1989). Its pulsation period is $6^{d} .17$; its orbital period is only $133^{d}$, a very low value for binary Cepheids (remember that classical Cepheids are supergiants). Even its velocity curve based on observations of a single year reveals quite obvious signatures of binarity.

We discovered the binarity of the classical Cepheids BY Cas, VY Cyg, VZ Cyg, and MW Cyg. Our results for more than 20 spectroscopic binary Cepheids, including the determination of their orbital elements, are summarized in Gorynya et al. (2000). The newest discovery is the spectroscopic binarity of V496 Aql (Fig. 2). For this star, we have two possible values for the orbital period, $1447^{d}$ and $573^{d}$; we tend to prefer the shorter one. Our observations 
show that at least $22 \%$ of the Cepheids are binaries; we consider the much higher estimates of some other authors, such as $50 \%$ or more, to be too high.

As noted above, our data are very advantageous for determinations of Cepheid radii using the Baade-Wesselink technique. From our original radii of 62 Cepheids, we have derived the period-radius relation in the form (Sachkov et al. 1998):

$$
\log R=1.23( \pm 0.03)+0.62( \pm 0.03) \log P .
$$

The radii derived with the Baade-Wesselink technique are very important as a tool to distinguish between different pulsation modes for Cepheids.

A relation between the light curve shape of a Cepheid and its period, the Hertzsprung sequence, is rather well known, but we could confidently reveal it, for the first time, in their radial velocity curves rather than in their light curves (Gorynya 1998).

In the recent years, we have entered into a fruitful collaboration with Dr. P. Moskalik (Warsaw) who uses our data for Fourier decomposition of radial velocity curves. This sensitive tool of research makes it possible to study the Hertzsprung sequence as well as to reveal resonances between different pulsation modes. Currently, Dr. Moskalik is studying our most recent observations and gives us advice on our program of observations, indicating insufficient coverage of velocity curves for stars of interest, hints to binarity, etc. We are planning to continue this cooperation. Also, we are trying to apply our instrument to the study of different variability types, but this is outside the scope of this presentation.

Acknowledgments. We are grateful to the astronomers who took part in the observations of our program, in particular, to Drs. S. Antipin, A. Rastorgouev, and M. Sachkov.

Our study was financially supported, in part, by grants from the Russian Foundation for Basic Research, the Program of Support for Leading Scientific Schools of Russia, and the Russian Federal Scientific and Technological Program "Astronomy".

\section{References}

Antipin, S.V. 1997, IBVS No. 4485

Antipin, S.V., Gorynya, N.A., Sachkov, M.E. et al. 1999, IBVS No. 4718

Baranne, A., Mayor, M., \& Poncet, J.L. 1979, Vistas in Astronomy 23, 279

Felgett, P.B. 1953, Optica Acta 2, 9

Gorynya, N.A. 1998, IBVS No. 4636

Gorynya, N.A., Samus, N.N., Sachkov, M.E. et al. 2000, in: The Impact of Large-Scale Surveys on Pulsating Star Research, L. Szabados and D.W. Kurtz (eds.), ASP Conference Series, 203, 242

Griffin, R.F. 1967, ApJ 148, 465

Harris, H.C. \& Welch, D.L. 1989, AJ 98, 981

Sachkov, M.E., Rastorguev, A.S., Samus, N.N., \& Gorynya, N.A. 1998, Astronomy Letters 24,377

Tokovinin, A.A. 1987, Soviet Ast. 31, 98 

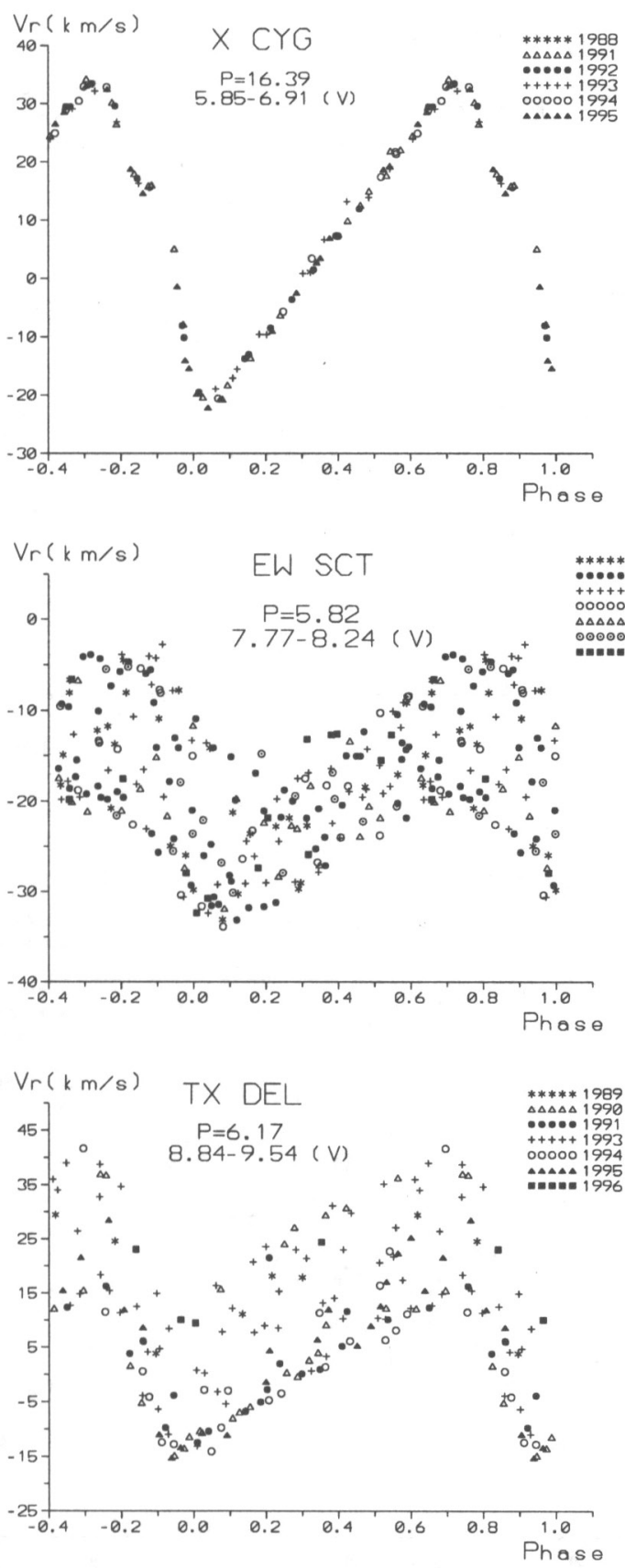

Figure 1. Radial velocity curves for three Cepheids, folded with their pulsation periods. EW Sct is a double-mode Cepheid, and TX Del is a spectroscopic binary. 

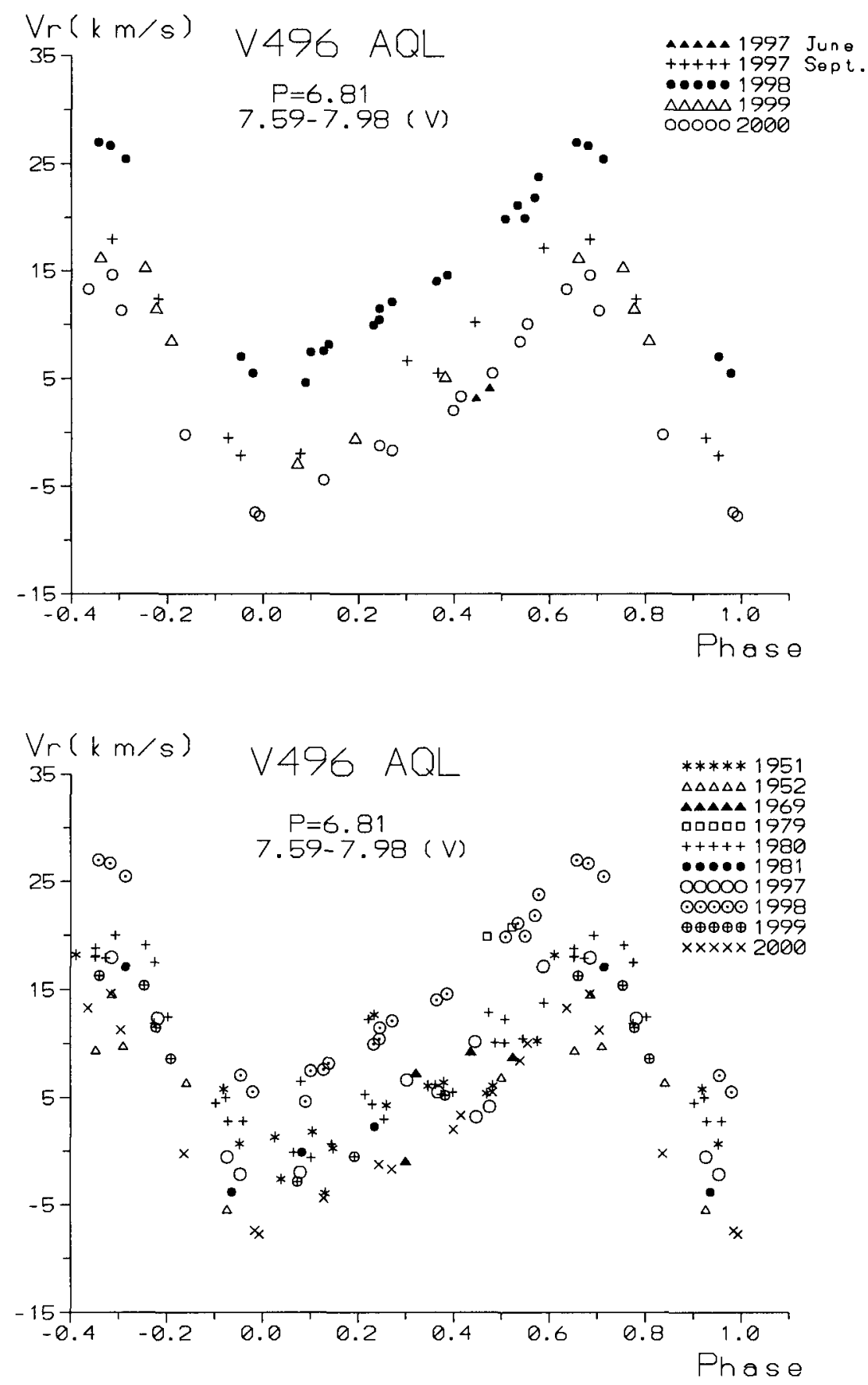

Figure 2. Radial velocities of $\mathrm{V} 496 \mathrm{Aql}$, plotted with the period of pulsations. 